MATEC Web of Conferences 22,02015 (2015)

DOI: $10.1051 /$ matec conf/20152202015

(C) Owned by the authors, published by EDP Sciences, 2015

\title{
A Study on a Miniaturized Planar Spiral Antenna for Partial Discharge Detection in GIS
}

\author{
Yongqiang Wang, Jianfang Li, Changyuan Li, Baolong Ouyang \& Zhihong Zheng \\ Hebei Provincial Key Laboratory of Power Transmission Equipment Security Defense, North China Electric \\ Power University, Baoding, Hebei, China
}

\begin{abstract}
Ultra-high frequency (UHF) detection method can effectively detect the signals of partial discharge (PD) in electrical equipment. Hence the authors designed a sensor of miniaturized planar spiral antenna for detecting PD in gas insulated substations (GISs), which is sine-wave meandered based on the traditional Archimedean spiral antenna in order to reduce the antenna's size. The test results indicate that this antenna has a wide-frequency band of $0.92 \sim 3 \mathrm{GHz}$ in terms of the voltage standing wave ratio (VSWR) which is smaller than 2. The antenna is smaller and its structure is simpler, its diameter is $97 \mathrm{~mm}$ and its height is $51 \mathrm{~mm}$; Besides, this antenna has an ultra-wideband, great directivity and the feature of omnidirectional radiation. Therefore, the proposed antenna is quite promising for PD on-line detection applications.
\end{abstract}

Keywords: ultra-high frequency; GIS; miniaturized planar spiral antenna; sine-wave meander; ultra-wideband

\section{INTRODUCTION}

In recent years, gas insulated substation (GIS) has been widely used in many large and important power plants. The statistical data of the CIGRE 23.10 working group's international report shows that, GIS insulation failure is mainly caused by partial discharge (PD) due to a variety of internal insulation defects. PD current pulse has a very fast rising edge, which can excite electromagnetic signals in the band of ultra-high frequency (UHF which is between $0.3 \sim 3 \mathrm{GHz}$ ) Therefore, we can choose the UHF detection method as a means of detecting GIS partial discharge.

The key technology of UHF detection method is to develop the UHF sensors. According to the different way of installation, the UHF sensors can be divided into the internal sensors and the external sensors. The internal sensors are placed in the GIS's cavity, so their anti-interference ability is strong, and their detection sensitivity is high. However, they cannot be put into the GIS devices which have been put into operation. At present, the study of internal sensors is mainly a combination of capacitive coupling principle and antenna theory, such as circular plate, cone and ring-type sensors and so on. External sensors can be installed on the insulating flange of GIS, which has been put into operation. To achieve the purpose of detecting GIS insulation condition, external sensors can collect UHF signals leaked through the insulating flange. The advantage of external sensors is that they don't affect the electric field inside the equipment, and it is very convenient to install external sensors. The disadvantages are their large structure, weak anti-interference ability and low sensitivity. At present, domestic scholars have launched a series of studies on external antenna sensors, including spiral antennas, ultra-wideband dipole antennas, the external micro-strip patch antenna (MPA), quasi-TEM horn antenna and fractal antennas and so on. However, as the UHF antennas' center frequency is low, it is hard to have antennas miniaturized. Only by increasing the size of the antenna can we reduce the resonant frequency. However, large antennas are unsuitable for application.

In recent years, scholars at home and abroad have done a lot of work to study the ultra-wideband miniaturized planar spiral antenna, but most ultra-wideband antennas are working at more than $2 \mathrm{GHz}$, working in small UHF ultra-wideband of miniaturized planar spiral antennas that have not been studied. To meet the requirement of UHF detection method for GIS, this paper designed a miniaturized planar spiral antenna, which has simple structure, small size, and wide-band features, and it is easy to install. The proposed antenna can effectively detect PD signals in the GIS.

\section{TRADITIONAL ARCHIMEDEAN SPIRAL} ANTENNA

Since the 1950s, the planar Archimedean spiral antenna has been proposed. Because of its broadband, circular polarization, low profile and other advantages, it is widely used in satellite communication and personal mobile communication. Its curve equation is shown as follows:

$r=r_{0}+a \varphi$

$(r, \varphi)$ is the polar coordinates of an arbitrary point on the curve; $\left(\mathrm{r}_{0}, 0\right)$ is the starting point; $a$ is the spiral growth rate. 


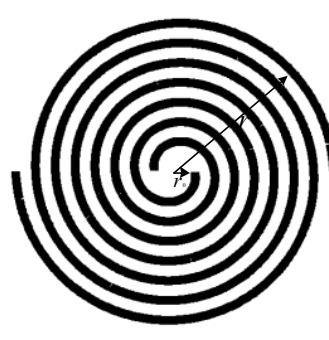

(a)

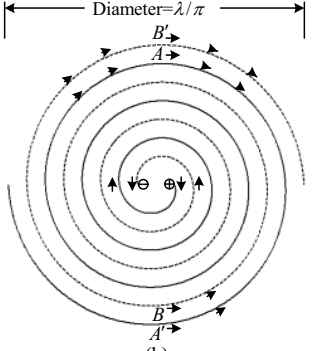

(b)
Figure 1. Planar Archimedean spiral antenna and its principle

As shown in Figure 1 (b), antenna arms are anti-phase fed at the starting point, A and A' represent opposite current at $\lambda / \pi$ diameter, and arc $\mathrm{AA}^{\prime}$ is about $\lambda / \pi$ long, so there is $180^{\circ}$ phase difference between the two arms, resulting in the formation of the same radiation. Because the current element point $\mathrm{B}$ on one arm and the current element point $\mathrm{A}$ on the other arm are in the same direction, and they have the same length from each starting point, they have the same phase. Similarly, the current element point B' and the current element point $\mathrm{A}^{\prime}$ are also in the same direction and the same phase. Such currents can form the strongest radiation on the spiral arms, when the diameter of the spiral arms is approximately equal to $\lambda / \pi$. That is to say, when the circumference is about one wavelength corresponding to the current frequency, it is the planar Archimedean spiral antenna's main radiation field ${ }^{[1]}$.

The spiral inner diameter $2 \mathrm{r}_{0}$ affects the maximum operating frequency and the feeding performance, it is shown as follows:

$$
2 r_{0} \leq \lambda_{h} / 4
$$

$\lambda_{\mathrm{h}}$ is the shortest wavelength.

Spiral outer diameter $2 r_{M}$ depends on the lowest operating frequency corresponding to the wavelength $\lambda_{1}$, it is shown as follows:

$$
2 \pi r_{M} \geq 1.25 \lambda_{l}
$$

Because the traditional planar Archimedean spiral antenna's outer diameter $2 r_{M}$ depends on the wavelength $\lambda_{1}$ of the low frequency, resulting in the antenna's size is very large ${ }^{[2]}$, which is quite contrary to the requirement of GIS miniaturized antenna for UHF PD detection. Therefore, it has very practical significance to design the miniaturized spiral antenna.

\section{CHARACTERISTICS OF MINIATURIZED PLANAR SPIRAL ANTENNA}

\subsection{Antenna's structure}

This paper designed a sine-wave meandered planar spiral antenna which is working in the range of
$0.9 \sim 3 \mathrm{GHz}$. In the specific design process, in order to achieve a good performance, we not only designed the antenna, but also designed the feeding part-Balun.

(1) Sine wave meandered planar spiral antenna

The traditional planar Archimedean spiral antenna's radiation mainly emerged when the radiation diameter is an integral multiple wavelength corresponding to the working frequency point. Through doing the same winding process to the spiral antenna's two arms, we can extend the effective electrical length and reduce the working frequency of the radiation field under the condition of radiation field is invariant ${ }^{[3]}$. Besides, as a result of the current phase characteristics between two adjacent arms remain unchanged, the winding process does not affect the antenna's radiation patterns and gain characteristics. At present, it is widely used to do some winding processes on antennas' arms with sine wave, square wave and triangle wave.

The outer spiral arm's length depends on the low frequency's wavelength, while the inner spiral arm's length depends on the antenna's maximum working frequency's wavelength ${ }^{[4]}$. In order to make the antenna's bandwidth relatively and evenly move to the low frequency and guarantee the high frequency characteristics of the antenna at the same time, this paper designed a sine wave meandered planar spiral antenna, whose inner eight laps still adopted the standard Archimedean spiral antenna. From the ninth circle, we began do sine-wave meander to spiral arms. In addition, in order to obtain the independent input impedance and match the broadband impedance smoothly, this paper adopted the self-complementary structure ${ }^{[5]}$, the spiral width and distance between the two spirals are equal. The designed sine wave meandered planar spiral antenna is shown in Figure 2.

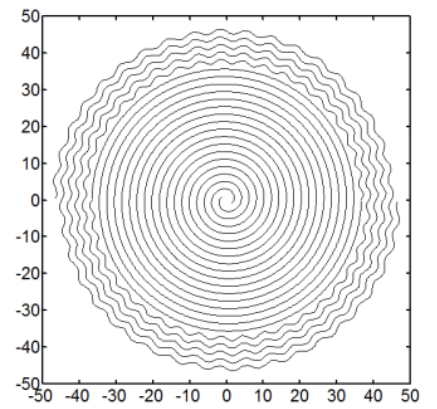

Figure 2. Planar spiral antenna with sine-wave meandered arms

In this antenna structure, the inner diameter $2 r_{0}$, spiral arms' width $\mathrm{w}$, sine wave amplitude $\mathrm{m}$ and each spiral line's sine wave cycle number $\mathrm{n}$ are the key factor to affect the antenna's radiation performance and miniaturization degree. In order to realize miniaturization, we should properly select these parameters based on the performance of the antenna. Compared with the antenna's simulating radiation characteristics from various cases, the parameters are shown as fol- 
lows: The spiral coil number is 11 ; the spiral growth rate $a=0.65$; the inner diameter $2 \mathrm{r}_{0}=4 \mathrm{~mm}$; the outer diameter $2 \mathrm{r}_{\mathrm{M}}=95.2 \mathrm{~mm}$; the spiral width $\mathrm{w}$ and distance between two spirals are $1 \mathrm{~mm}$; the sine-wave amplitude $\mathrm{m}=0.6 \mathrm{~mm}$; the sine-wave cycle number $\mathrm{n}=40$; the input impedance is $120 \Omega$.

The antenna model is shown in Figure 3, the antenna's radiation surface is printed on a dielectric substrate with thickness of $1 \mathrm{~mm}$, and the dielectric substrate material is FR4 whose dielectric constant is 4.4.

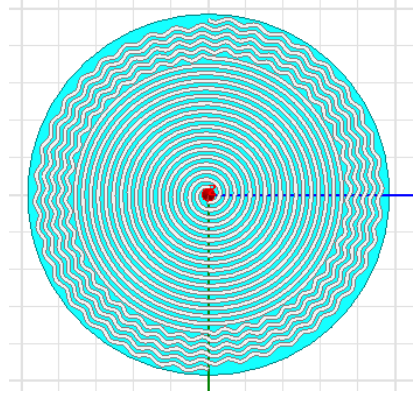

Figure 3. Antenna

(2) The design of Balun

Because the planar Archimedean spiral antenna is a symmetrical balance structure, it needs a balanced feeder to feed the spiral antenna. However, the traditional feeding mode - coaxial cable is non-balanced, if the coaxial cable is used for feeding the antenna, we need to transform the balanced impedance of the antenna to the unbalanced impedance of the Balun ${ }^{[6]}$. Besides, the antenna has the broad-band characteristic, thus, the Balun-design should also has the broad-band characteristic.

In this paper, we used an exponential taper micro-strip Balun, which can be fed with the coaxial cable as shown in Figure 4. The floor and the micro-strip line are used in the form of exponential gradient, which makes the Balun's unbalanced micro-strip line structure be gradually transferred to the balance feeder structure, realizing the unbalanced input $50 \Omega$ impedance to the output $120 \Omega$ impedance transformed in the working frequency band ${ }^{[7]}$.

In this paper, the designed Balun is printed on the FR4 medium substrate, the substrate's thickness is $2 \mathrm{~mm}$ and its dielectric constant is 4.4. The Balun's length is $50 \mathrm{~mm}$ and its width is $26 \mathrm{~mm}$. The Balun is shown in Figure 4.

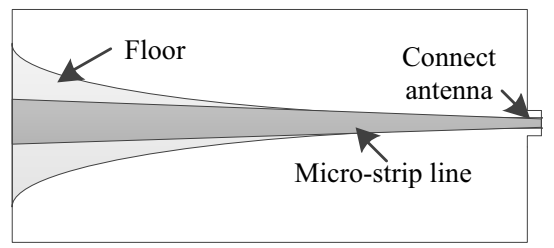

Figure 4. Schematic diagram of the structure of the exponential transition micro-strip Balun

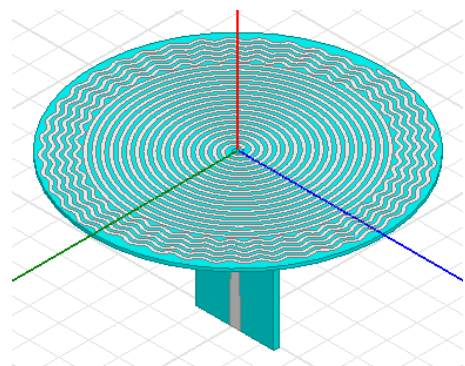

Figure 5. Model of the sine wave meandered planar spiral antenna

\subsection{VSWR}

The antenna's input impedance is the feeder's load impedance. The matching degree between the antenna's input impedance and the coaxial cable's output impedance will directly determine the antenna's input and output energy efficiency ${ }^{[8]}$. In rare cases, its value can be calculated by using strict theory. It is commonly used that the voltage standing wave ratio (VSWR) can show the matching degree between the input impedance and the coaxial cable's impedance. The ideal matching state is acquired when the voltage standing wave ratio $($ VSWR $)=1$. However, in fact, it is impossible to achieve. When VSWR is less than 2, the power the antenna transfers will be achieved more than $88.9 \%$. In general engineering, the voltage standing wave ratio which is less than 2 is required.

In this paper, we use ANSYS HFSS to establish the model of the antenna with the Balun, within the frequency range from $0.3 \mathrm{GHz}$ to $3 \mathrm{GHz}$, the simulated results of VSWR curve is shown in Figure 6. As it can be seen from the graph, in the $1.1 \sim 3 \mathrm{GHz}$ frequency range, the VSWR is less than 2.

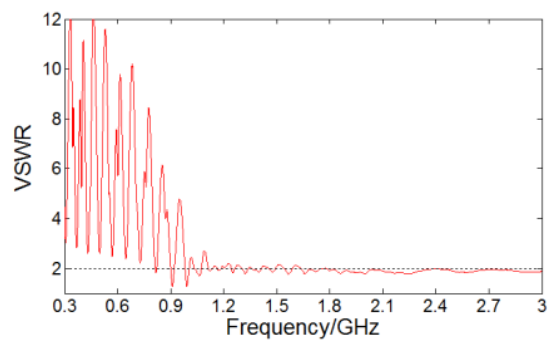

Figure 6. Simulated VSWR curve of the antenna

After the antenna is completed, we used the vector network analyzer to test VSWR of the antenna, the sweep range of $0.3 \sim 3 \mathrm{GHZ}$ and the measured VSWR curve are shown in Figure 6. The test result shows that the frequency range of $0.92 \sim 3 \mathrm{GHZ}$ is ideal when VSWR is less than 2, and the antenna's performance is good. But when it is below $630 \mathrm{MHZ}$, VSWR is bad. Compared Figure 4 with Figure 5, it can be seen that the simulated results and the measured results are 
basically same.

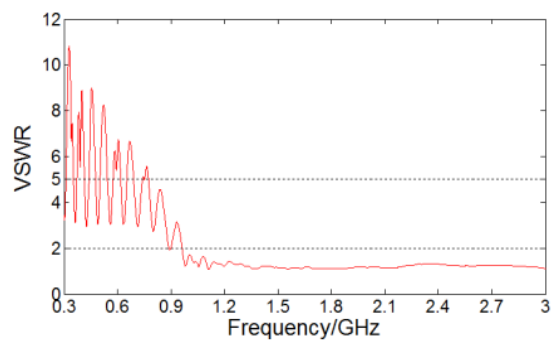

Figure 7. Measured VSWR curve of the antenna

\subsection{Antenna's gain and radiation pattern}

Because the spiral antenna has axial rotation symmetry characteristic, this article only tested antenna's radiation pattern at the frequency point of $0.3 \mathrm{GHz}$, $0.5 \mathrm{GHz}, 0.8 \mathrm{GHz}, 1 \mathrm{GHz}, 1.5 \mathrm{GHz}, 2 \mathrm{GHz}, 2.5 \mathrm{GHz}$ and $3 \mathrm{GHz}$, as shown in following figures. The figures show that the antenna has great directivity and omnidirectional radiation characteristics in the whole frequency range. In the whole frequency range, $\mathrm{H}$ plane is "eight" shape at the frequency below $500 \mathrm{MHz}$, and the $\mathrm{E}$ plane is a regular round. In the frequency range of $800 \mathrm{MHZ} \sim 3 \mathrm{GHZ}$, the E plane is "eight" shape. With the increase of frequency, the performance of the antenna gradually becomes worse, and the radiation pattern becomes distorted. The possible reason is that with the increase of frequency, the antenna can receive and radiate higher radiation of the electromagnetic wave.

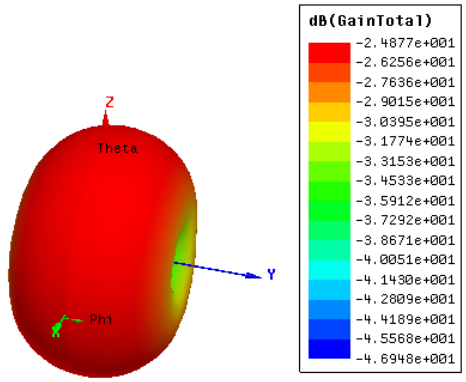

(a)

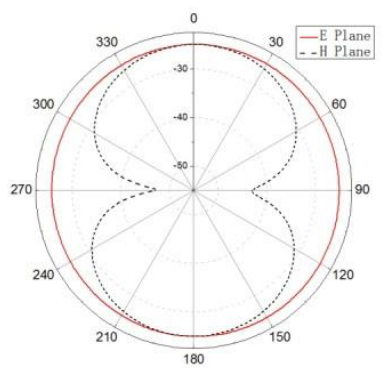

(b)

Figure 8. (a) $3 \mathrm{D}$ radiation pattern of the antenna when the frequency is $0.3 \mathrm{GHz}$; (b) Radiation patterns in the $\mathrm{XZ} \& \mathrm{YZ}$ plane when the frequency is $0.3 \mathrm{GHz}$.

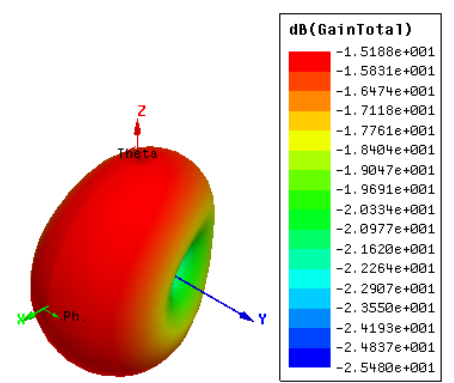

(a)

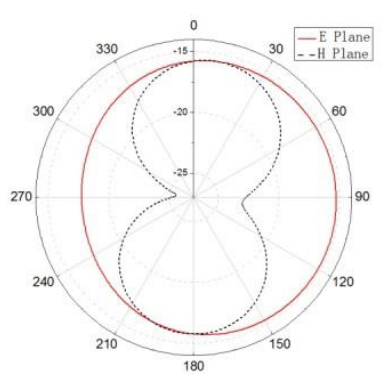

(b)

Figure 9. (a) 3D radiation pattern of the antenna when the frequency is $0.5 \mathrm{GHz}$; (b) Radiation patterns in the $\mathrm{XZ} \& \mathrm{YZ}$ plane when the frequency is $0.5 \mathrm{GHz}$.

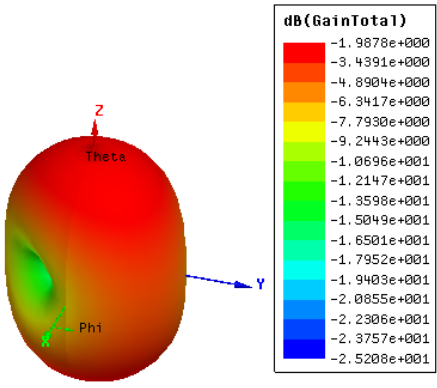

(a)

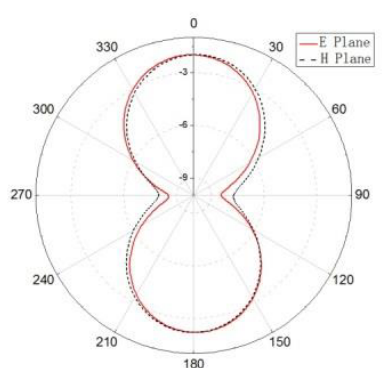

(b)

Figure 10. (a) 3D radiation pattern of the antenna when the frequency is $0.8 \mathrm{GHz}$; (b) Radiation patterns in the $\mathrm{XZ} \& \mathrm{YZ}$ 
plane when the frequency is $0.8 \mathrm{GHz}$.

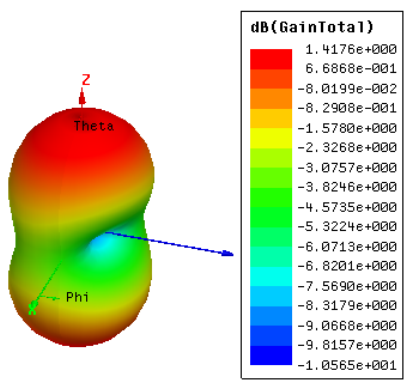

(a)

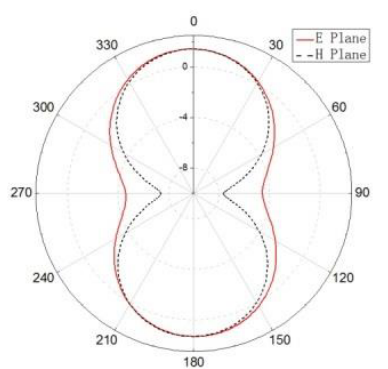

(b)

Figure 11. (a) 3D radiation pattern of the antenna when the frequency is $1 \mathrm{GHz}$; (b) Radiation patterns in the XZ\&YZ plane when the frequency is $1 \mathrm{GHz}$.

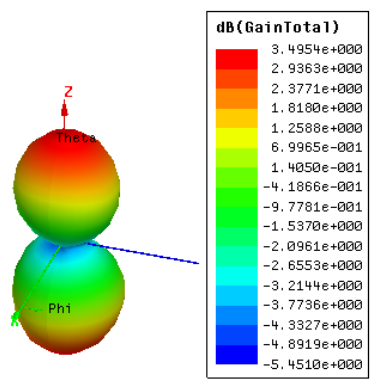

(a)

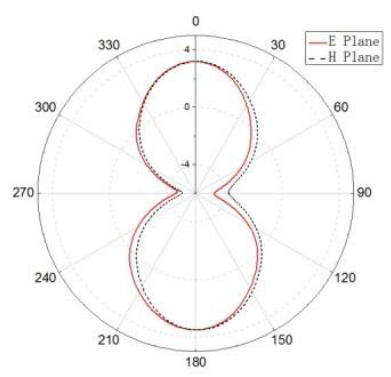

(b)
Figure 12. (a) 3D radiation pattern of the antenna when the frequency is $1.5 \mathrm{GHz}$; (b) Radiation patterns in the $\mathrm{XZ} \& \mathrm{YZ}$ plane when the frequency is $1.5 \mathrm{GHz}$.

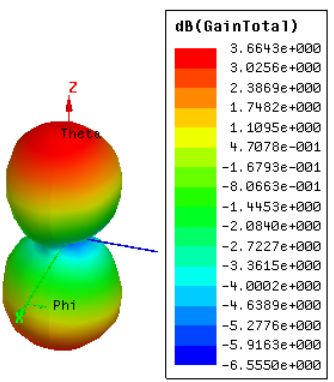

(a)

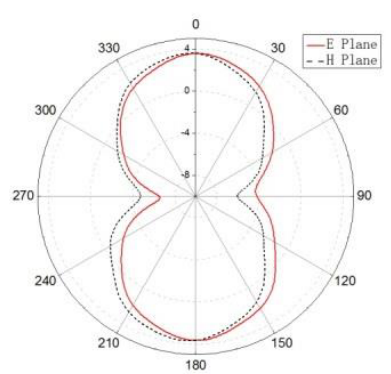

(b)

Figure 13. (a) $3 \mathrm{D}$ radiation pattern of the antenna when the frequency is $2 \mathrm{GHz}$; (b) Radiation patterns in the XZ\&YZ plane when the frequency is $2 \mathrm{GHz}$.

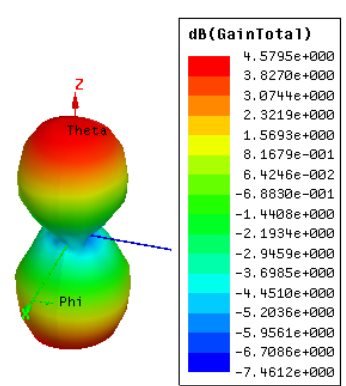

(a)

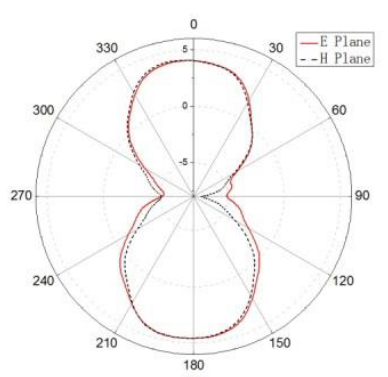

(b) 
MATEC Web of Conferences

Table 1. Testing records of gain

\begin{tabular}{lllllllll}
\hline Frequency/GHz & 0.3 & 0.5 & 0.8 & 1 & 1.5 & 2 & 2.5 & 3 \\
\hline Gain/dB & -24.9 & -15.2 & -2.0 & 1.4 & 3.5 & 3.7 & 4.6 & 5.1 \\
\hline
\end{tabular}

Figure 14. (a) 3D radiation pattern of the antenna when the frequency is $2.5 \mathrm{GHz}$; (b) Radiation patterns in the $\mathrm{XZ} \& \mathrm{YZ}$ plane when the frequency is $2.5 \mathrm{GHz}$.

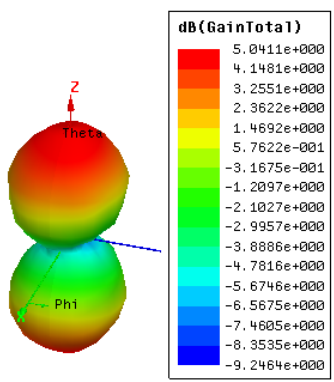

(a)

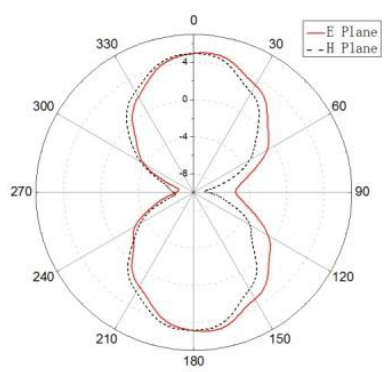

(b)

Figure 15. (a) 3D radiation pattern of the antenna when the frequency is $3 \mathrm{GHz}$; (b) Radiation patterns in the $\mathrm{XZ} \& \mathrm{YZ}$ plane when the frequency is $3 \mathrm{GHz}$.

The antenna's gain test results in the nine frequency point are shown in Table 1. Along with the increased frequency of electromagnetic radiation, the antenna's gain is up from $-24.9 \mathrm{~dB}$ to $5.1 \mathrm{~dB}$ when the frequency is from $0.3 \mathrm{GHz}$ to $3 \mathrm{GHz}$. The antenna can amplify the UHF weak signals, which is conducive to detect GIS partial discharge.

\section{CONCLUSION}

With the development of ultra-wideband technology, the conformal ultra-wideband antenna is a hot topic of research in the field of antenna technology. This paper discussed a kind of miniaturized planar Archimedean spiral antenna and simulated its radiation performance through the full-wave electromagnetic simulation software accurately. The structure and parameters of miniature planar spiral Archimedean antenna are designed and the simulation results show that the dis- cussed antenna has an ultra-wideband performance. The proposed miniaturized planar Archimedean spiral antenna can act as one kind of ultra-wideband antenna which can be selected to apply in GIS equipment for partial discharge detection.

\section{ACKNOWLEDGEMENT}

The authors would like to express their sincere gratitude to ANSYS Ltd for providing the HFSS Training Center at our university with a free package of the software.

This project is supported by National High-tech Research and Development Program of China (863 Program)(2012AA050802), and the Fundamental Research Funds for the Central Universities(13MS73)

\section{REFERENCES}

[1] Kramer B A, Chen C C, Volakis J L. 2008. Size reduction of a low-profile apiral antenna using inductive and dielectric loading. IEEE Antennas and Wireless Propagation Letters, 7: 22-25.

[2] Hisamatsu Nakano, Tatsuya Igarashi, Hiroshi Oyanagi, et al. 2009. Unbalanced-mode spiral antenna backed by an extremely shallow cavity. IEEE Transactions on Antennas and Propagation, 57(6): 1625-1633.

[3] Li Weizhong, Yang Gang. 2009. A new low profile Archimedean spiral antenna. Guidance \& Fuze, 30(3): 28-31.

[4] Xuan Xiaobo, Zhang Yuan. 2006. The design of antenna for microwave broadband passive fuze with phase compare. Guidance \& Fuze, 27(2): 47-50.

[5] Thorsten W. Hertel, Glenn S. Smith. 2002. Analysis and design of two-arm conical spiral antennas. IEEE transactions on electromagnetic compatibility, 44(1): 25-37.

[6] Chen Duo, Yuan Jiansheng. 2007. Numerical simulation of dispersive properties for conical spiral antenna used for pulse radiation. Chinese Journal of Radio Science. 22(4): 589-592.

[7] Shen Renqiang, Yin Yingzeng, Ma Jinping, Zhao Na. 2007. Analysis and design of conical printed quadrifilar helical antenna. Journal of Microwaves. 123(5): 14-18.

[8] Salam K. Kharmas. 2008. Moment method analysis of an Archimedean spiral printed on a layered dielectric sphere. IEEE Transactions on Antennas and Propagation, 56(2): 345-352. 